Argonne

\title{
A physics-informed data-driven low order model for the wind velocity deficit at the wake of isolated buildings
}

FY2021 Q2

Environmental Science Division and Computational Science Division 


\section{About Argonne National Laboratory}

Argonne is a U.S. Department of Energy laboratory managed by UChicago Argonne, LLC under contract DE-AC02-06CH11357. The Laboratory's main facility is outside Chicago, at 9700 South Cass Avenue, Argonne, Illinois 60439. For information about Argonne and its pioneering science and technology programs, see www.anl.gov.

\section{DOCUMENT AVAILABILITY}

Online Access: U.S. Department of Energy (DOE) reports produced after 1991 and a growing number of pre-1991 documents are available free at OSTI.GOV (http://www.osti.gov/), a service of the US Dept. of Energy's Office of Scientific and Technical Information.

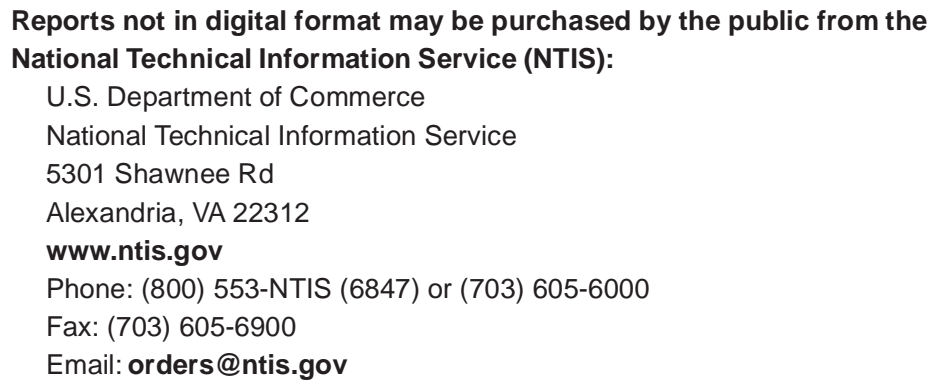

Reports not in digital format are available to DOE and DOE contractors from the Office of Scientific and Technical Information (OSTI):

U.S. Department of Energy

Office of Scientific and Technical Information

P.O. Box 62

Oak Ridge, TN 37831-0062

www.osti.gov

Phone: (865) 576-8401

Fax: (865) 576-5728

Email: reports@osti.gov

\section{Disclaimer}

This report was prepared as an account of work sponsored by an agency of the United States Government. Neither the United States Government nor any agency thereof, nor UChicago Argonne, LLC, nor any of their employees or officers, makes any warranty, express or implied, or assumes any legal liability or responsibility for the accuracy, completeness, or usefulness of any information, apparatus, product, or process disclosed, or represents that its use would not infringe privately owned rights. Reference herein to any specific commercial product, process, or service by trade name, trademark, manufacturer, or otherwise, does not necessarily constitute or imply its endorsement, recommendation, or favoring by the United States Government or any agency thereof. The views and opinions of document authors expressed herein do not necessarily state or reflect those of the United States Government or any agency thereof, Argonne National Laboratory, or UChicago Argonne, LLC. 


\section{A physics-informed data-driven low order model for the wind velocity deficit at the wake of isolated buildings}

FY2021 Q2

prepared by

Dimitrios K. Fytanidis*, Romit Maulik ${ }^{\dagger}$, Ramesh Balakrishnan*, and Rao Kotamarthi

* Computational Science Division, Argonne National Laboratory

† Argonne Leadership Computing Facility, Argonne National Laboratory

‡Environmental Science Science Division, Argonne National Laboratory

Prepared for

DOE EERE Wind Energy Technology Office

March 312021 


\section{Contents}

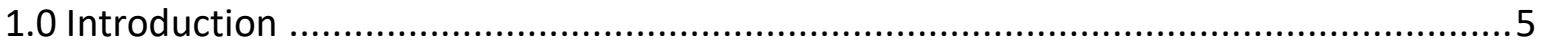

2.0 Simulations of flow around wall mounted cuboids: modeling results......................... 6

2.1 Comparison between numerical results and previous experimental and numerical .........

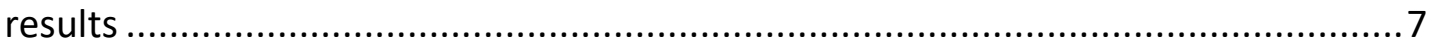

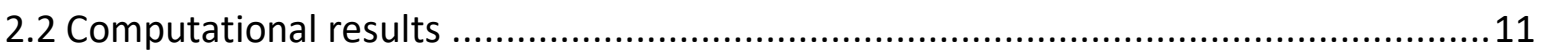

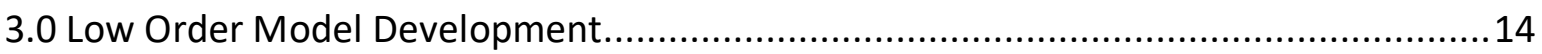

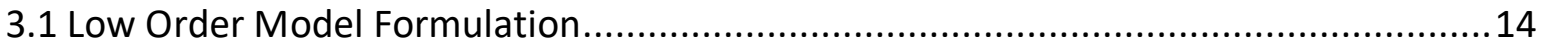

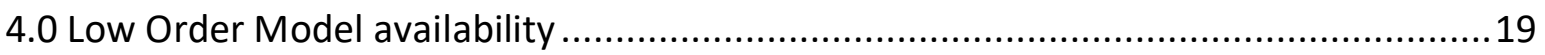

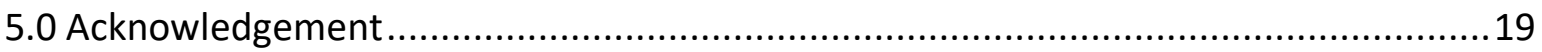

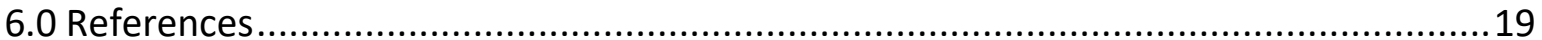




\subsection{Introduction}

Detailed simulations of the Atmospheric boundary layer (ABL) provides information on wind velocity, direction and turbulence for optimizing the design of wind turbines and wind farms and their operations. Distributed wind turbines have been considered for use in urban or suburban areas for covering energy needs using a sustainable energy solution. Simulations of high Reynolds number turbulent ABL flows past obstacles can be used for the prediction of the velocity deficit and turbulence characteristics in the leeward side of building-like obstacles, allowing for the estimation of power losses and fatigue loads in wind turbines. However, fully three-dimensional simulations of high Reynolds number ABL flows using Large Eddy Simulation (LES) or Reynolds Average Navier-Stokes Simulations (RANS) continue to require large computational/ execution times. As a result, these 3D simulations may not be ideal tools for the rapid assessment of wind energy potential. In the present study, 3D numerical results from wall resolved RANS simulations were used for the development of a low order model that allows the rapid assessment of wind velocity and turbulence in the leeward side of an isolated building.

In our current study we used computationally efficient Reynolds average Navier-Stokes simulations for the prediction of the complex flow patterns in the wake of cuboids which represent buildings. Specifically, the high-order spectral-element based solver Nek5000 (Fischer et al. 2008) was used for the solution of the governing equations. The wind flow in the results presented herein was assumed to be turbulent, and incompressible. Realistic boundary conditions were applied for the velocity and turbulence quantities to mimic the ABL flow conditions. The $k-t$ turbulence model (Speziale et al 1992) combined with the Boussinesque approximation was applied for the closure problem.

The simulation results were compared against experimental wind tunnel observations and previously done simulations performed using the finite volume code OpenFOAM. Additionally, wall-resolving simulation results were compared against wall-modelled results. For the wall-modeled case a new mixed no-slip/traction approach was adopted which allows the use of no-slip boundary condition at the sharp corners of the cuboids while traction boundary conditions was adopted in the rest of the surface. The effect of wind angle of attack on the wake formation was examined by creating several different computational domains. Also, the effect of different aspect ratios of the cuboids on the wake was examined.

Analytical models from the literature (Robins and Apsley 2020, Counihan et al. 1974, Kothari et al. 1979, and Peterka et al. 1985) developed for the case of an isolated cubes and zero wind angle of attack $\left(\theta=0.0^{\circ}\right)$ were generalized to take into consideration different angles of attack and the effect of different building aspect ratios. Using the validated CFD results and surrogate model techniques combined with the machine learning based algorithms included in the open-source package Tensorflow, a generalized model is proposed. Additionally, to tackle the known issues of the simple wake models (Kothari et al. 1979, and Peterka et al. 1985) to predict the acceleration of the wind flow around the buildings, an additional horseshoe-vortex/accelerated-flow correction function has been generalized to take into consideration different angles of attack and building aspect ratios. 
The proposed low-order model (LOM) is capable to predict the perturbation of an approaching/undisturbed flow profile $u$ at the wake of a cuboid representing a building (figure 1). The resulting velocity profile $u$ ' can be estimated using estimates of the perturbation function $f\left(f=u^{\prime}-u\right)$ based on the parameters $L, W$ and $H . L, W$ and $H$ are the streamwise, spanwise and vertical dimensions of the cube that is parallel to the flow and can enclose the actual cuboid with dimensions $L_{b} \times W_{b \times} H$ (length $\times$ width $\times$ height).

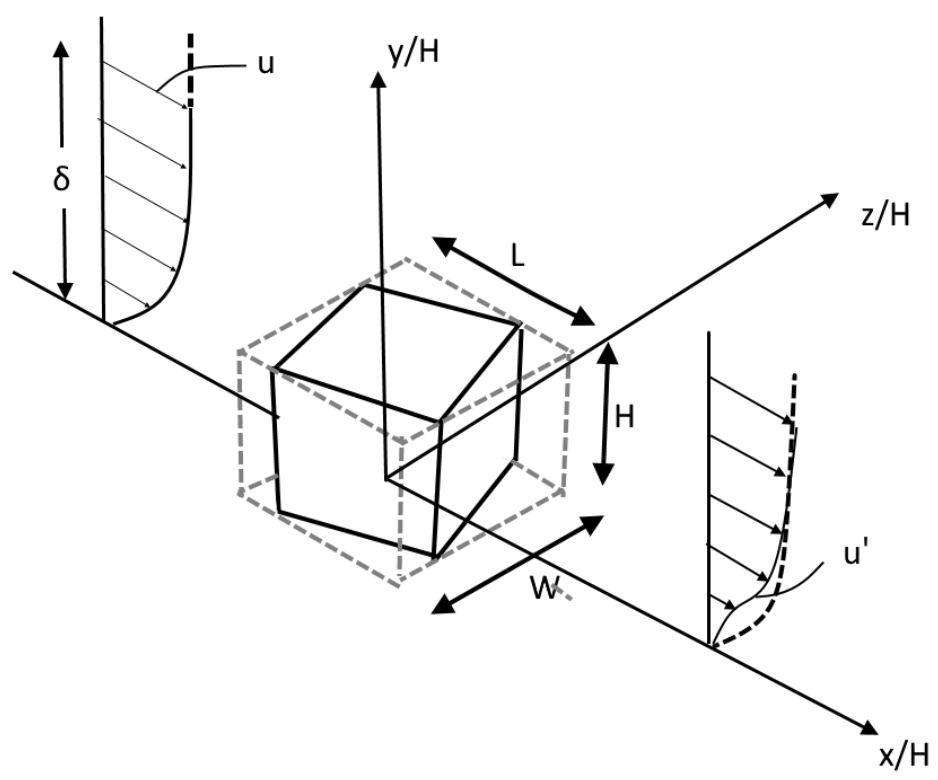

Figure 1 Schematic of the examined cases.

\subsection{Simulations of flow around wall mounted cuboids: modeling results}

Simulations of the turbulent flow around different cuboids representing building of various aspect ratios were conducted using the Reynolds Average Navier-Stokes (RANS) approach. Different angles of attack were also considered resulting to a wide range of $L, W$, and $H$ (and $L_{b \times} W_{b \times} H$ ) values. A summary of the examined cases is shown in table 1 . The Reynolds number based on the height of the building $H$ and the velocity at the height of the building $U_{H}$ was assumed to be $\operatorname{Re}_{H}=\frac{U_{H} H}{v}=40,000$ (where $v$ is the kinematic viscosity of the air). The examined domain size was $31.5 \mathrm{H}$ long and $6.8 \mathrm{H}$ wide. The height of the domain was varied and equal to $5 \mathrm{H}, 6 \mathrm{H}$, and $7 \mathrm{H}$ for the cases with building heigh $1 \mathrm{H}, 2 \mathrm{H}$, and $3 \mathrm{H}$ respectively.

For the estimation of Reynolds stresses a two-equation $k$-T closure model was adopted. Realistic profiles for the turbulence quantities $k$ and $\mathrm{T}$ were adopted for the inlet in addition to the imposed fully developed logarithmic profile for the velocity. The top surface of the computational domain was assumed to be stress-free. For the spanwise surfaces of the computational domain a periodic boundary condition is adopted. The terrain and cuboid surfaces were assumed to be solid walls. To simulate the wall effects two different approaches were examined, a wall resolving approach for which a no-slip 
boundary condition is imposed at the wall (combined with $k=T=0.0$ boundary condition for the turbulence quantities) and a wall-function/traction boundary condition which uses the equilibrium logarithmic profile for the estimation of shear stresses at the wall and some equilibrium expressions for $k$ and $t$.

The governing equations were solved using the spectral-element based solver nek5000 (Fischer et al. 2008). The simulations results in the present analysis were conducted using 7th-order elements ( $\mid x 1=8)$ to maximize both spatial accuracy and computational efficiency for fast convergence of the simulations. All the liner terms were treated implicitly with pressure/velocity decoupling (Deville et al. 2002, Fischer et al. 2008) while the nonlinear advection terms were treated explicitly using third order BDF3/EXT3 schemes.

Table 1 Cases used for the tuning of the low order method

\begin{tabular}{|c|c|c|c|c|c|c|}
\hline $\begin{array}{l}\text { Case name } \\
L_{b} \times W_{b} \times H-\theta\end{array}$ & $\begin{array}{l}\text { dimensionless } \\
\text { height } \mathrm{H}\end{array}$ & $L_{b}$ & $W_{b}$ & $\begin{array}{l}\text { wind angle } \\
\text { of attack } \theta\end{array}$ & $\begin{array}{l}\text { dimensionless } \\
\text { projected } \\
\text { length } L\end{array}$ & $\begin{array}{l}\text { dimensionless } \\
\text { projected width W }\end{array}$ \\
\hline $1 \times 1 \times 1-0.0$ & 1.0 & 1.0 & 1.0 & $0.0^{\circ}$ & 1.000 & 1.000 \\
\hline $1 \times 1 \times-11.25$ & 1.0 & 1.0 & 1.0 & $11.25^{\circ}$ & 1.176 & 1.176 \\
\hline $1 \times 1 \times 1-22.5$ & 1.0 & 1.0 & 1.0 & $22.5^{\circ}$ & 1.307 & 1.307 \\
\hline $1 \times 1 \times 1-33.76$ & 1.0 & 1.0 & 1.0 & $33.75^{\circ}$ & 1.387 & 1.387 \\
\hline $1 \times 1 \times 1-45.0$ & 1.0 & 1.0 & 1.0 & 45.0 & 1.414 & 1.414 \\
\hline $1 \times 1 \times 2-0.0$ & 2.0 & 1.0 & 1.0 & $0.0^{\circ}$ & 1.000 & 1.000 \\
\hline $1 \times 1 \times 2-11.25$ & 2.0 & 1.0 & 1.0 & $11.25^{\circ}$ & 1.176 & 1.176 \\
\hline $1 \times 1 \times 2-22.5$ & 2.0 & 1.0 & 1.0 & $22.5^{\circ}$ & 1.307 & 1.307 \\
\hline $1 \times 1 \times 2-33.76$ & 2.0 & 1.0 & 1.0 & $33.75^{\circ}$ & 1.387 & 1.387 \\
\hline $1 \times 1 \times 2-45.0$ & 2.0 & 1.0 & 1.0 & 45.0 & 1.414 & 1.414 \\
\hline $1 \times 1 \times 3-0.0$ & 3.0 & 1.0 & 1.0 & $0.0^{\circ}$ & 1.000 & 1.000 \\
\hline $1 \times 1 \times 3-11.25$ & 3.0 & 1.0 & 1.0 & $11.25^{\circ}$ & 1.176 & 1.176 \\
\hline $1 \times 1 \times 3-22.5$ & 3.0 & 1.0 & 1.0 & $22.5^{\circ}$ & 1.307 & 1.307 \\
\hline $1 \times 1 \times 3-33.76$ & 3.0 & 1.0 & 1.0 & $33.75^{\circ}$ & 1.387 & 1.387 \\
\hline $1 \times 1 \times 3-45.0$ & 3.0 & 1.0 & 1.0 & 45.0 & 1.414 & 1.414 \\
\hline $1 \times 2 \times 1-0.0$ & 1.0 & 1.0 & 2.0 & $0.0^{\circ}$ & 1.000 & 2.000 \\
\hline $1 \times 2 \times 1-22.5$ & 1.0 & 1.0 & 2.0 & $22.5^{\circ}$ & 1.689 & 2.230 \\
\hline $1 \times 2 \times 1-45.0$ & 1.0 & 1.0 & 2.0 & $45.0^{\circ}$ & 2.121 & 2.121 \\
\hline $1 \times 2 \times 1-67.5$ & 1.0 & 1.0 & 2.0 & $67.5^{\circ}$ & 2.230 & 1.689 \\
\hline $1 \times 2 \times 1-90.0$ & 1.0 & 1.0 & 2.0 & 90.0 & 2.000 & 1.000 \\
\hline
\end{tabular}

\subsection{Comparison between numerical results and previous experimental and numerical results}

The numerical results from the simulations performed using Nek5000 were initially compared against previous simulations performed using the second-order finite volumebased solver OpenFOAM and experimental observation by Snyder and Lawson (1994). Figure 2 shows the comparison between Nek5000 with "wall resolving" boundary condition against the results using "wall functions" for the case of a wall mounted cuboid with dimensions $1 \times 1 \times 1$ and wind angle of attack $\theta=0^{\circ}$. The observations by Snyder and Lawson (1994) are also plotted for comparison. The agreement between the two 
approaches is good, suggesting that the applied resolution is adequate to resolve the wall surfaces.

More detailed comparison of the Nek5000 simulations results against the experimental observations and previous OpenFOAM simulations are shown in figures 3 and 4 . It is important to note here that for the OpenFOAM simulations a $k-\varepsilon$ turbulence closure and "fully rough wall functions" were adopted, while for the Nek5000 simulations smooth walls were either resolved or modeled using a new mixed no-slip/traction approach. The mixed no-slip/traction approach allows the use of no-slip boundary condition at the sharp corners and a traction boundary condition elsewhere. The results in figure 2 and figures 3 and 4 show that the wall resolving and the wall modeled approach using Nek5000 give practically identical results. Additionally, no significant difference in the accuracy of Nek5000 and OpenFOAM was observed. In general, Nek5000 results are close to the experimental observations by Snyder and Lawson (1994).

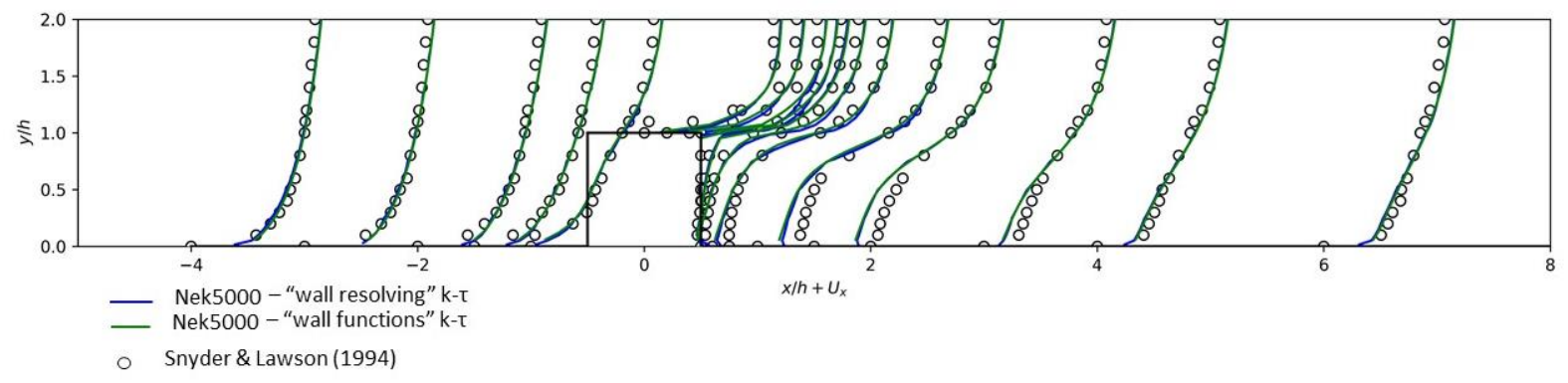

Figure 2 Comparison between wall resolving and wall modeled cases. 

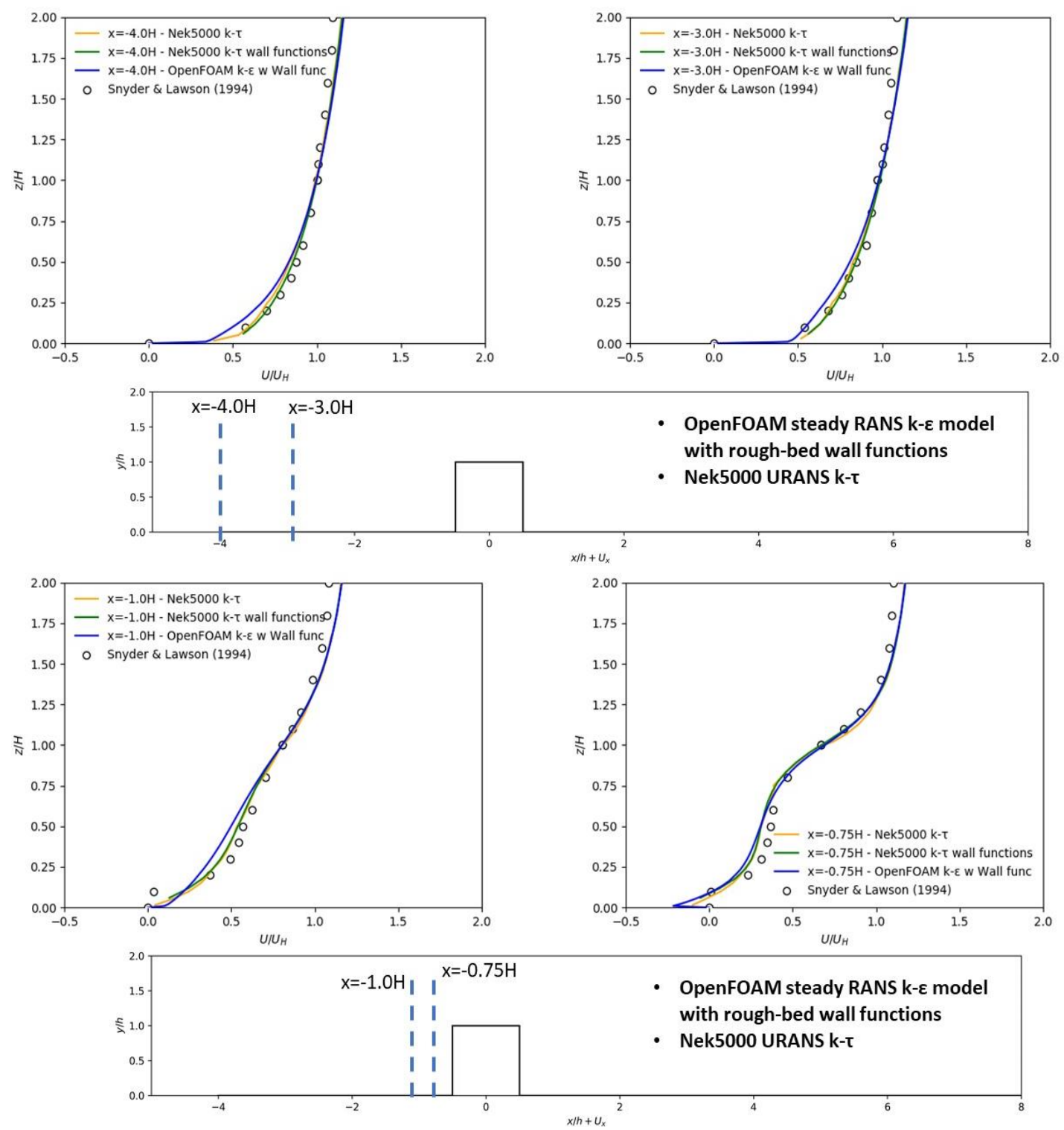

Figure 3 Numerical results for the case of $L_{b} \times W_{b} x H=[1 \times 1 \times 1]$. Nek5000 wall resolving and wall modeled simulations comparison against OpenFOAM simulation results and Snyder and Lawson (1994) measurements $(x=-4.0 H$, $3.0 H,-1.0 H$, and $-0.75 H$ ). 

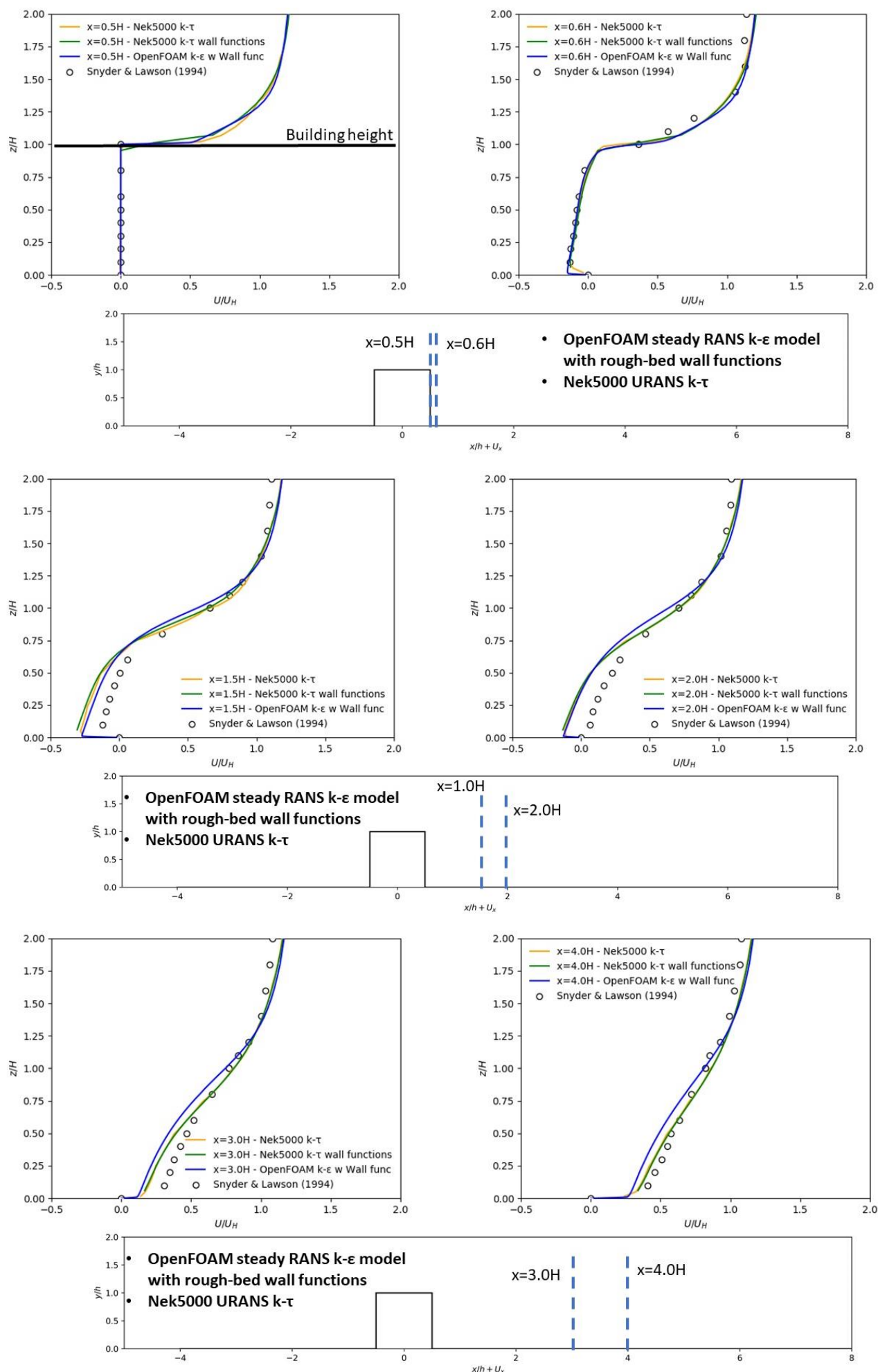

Figure 4 Numerical results for the case of $L_{b} \times W_{b} \times H=[1 \times 1 \times 1]$. Nek5000 wall resolving and wall modeled simulations comparison against OpenFOAM simulation results and Snyder and Lawson (1994) measurements $(x=0.5 H, 0.6 H$, $1.0 H, 2.0 H, 3.0 H$, and $4.0 H$ ) 


\subsection{Computational results}

In the present paragraph some results from the parametric analysis presented in table 1 are presented. Figure 5 shows the wake formation for a cuboid with dimensions $L_{b} \times W_{b} \times H=[1 H \times 1 H \times 1 H]$. All the boundary conditions and flow conditions are kept identical between the different simulations. In addition to the velocity magnitude results in perspective view, velocity magnate results are plotted on slices at a heigh of $0.1 \mathrm{H}$. The results show that angle of attack significantly alters the wake structure and recirculation length $L_{r}$.

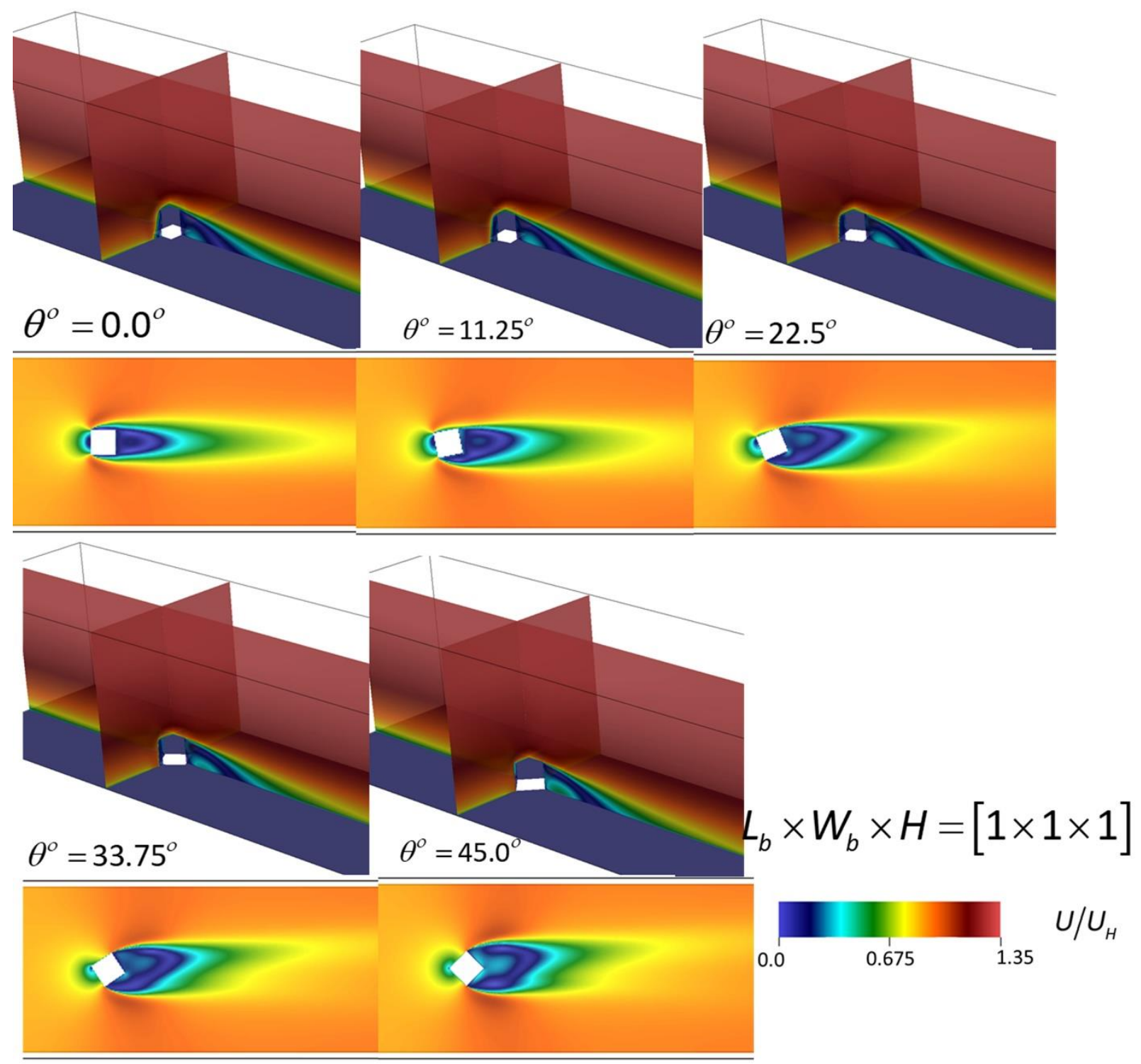

Figure 5 Wake results for $L_{b} \times W_{b} \times H=[1 \times 1 \times 1]$ for various angles of wind attack (Top: perspective views, Bottom: slides at $\mathrm{z}=0.1 \mathrm{H}$ ) 
Similar results for the case of a cuboid with dimensios $L_{b} \times W_{b} \times H=[1 H \times 1 H \times 2 H]$ are presented in figure 6 . Comparing the results in figure 5 and 5 we can see that the heigh of the building significantly alters the wake and the recirculation zone characteristics. This effect is consistent for taller cuboids. Figure 7 shows this effect on a slide at $z=0.1 \mathrm{H}$. The recirculation length at the centerline of the domain $(\mathrm{y}=0.0)$ for cuboids with dimensions $1 \mathrm{H} \times 1 \mathrm{H} \times 1 \mathrm{H}\left(\mathrm{L}_{b} \times W_{b} \times H\right), 1 \mathrm{H} \times 1 \mathrm{H} \times 2 \mathrm{H}$ and $1 \mathrm{H} \times 1 \mathrm{H} \times 3 \mathrm{H}$ for all the angles of attack are plotted in figure 8 . It is shown that the recirculation zone's length increases for higher values of projected length $L$ and taller buildings.
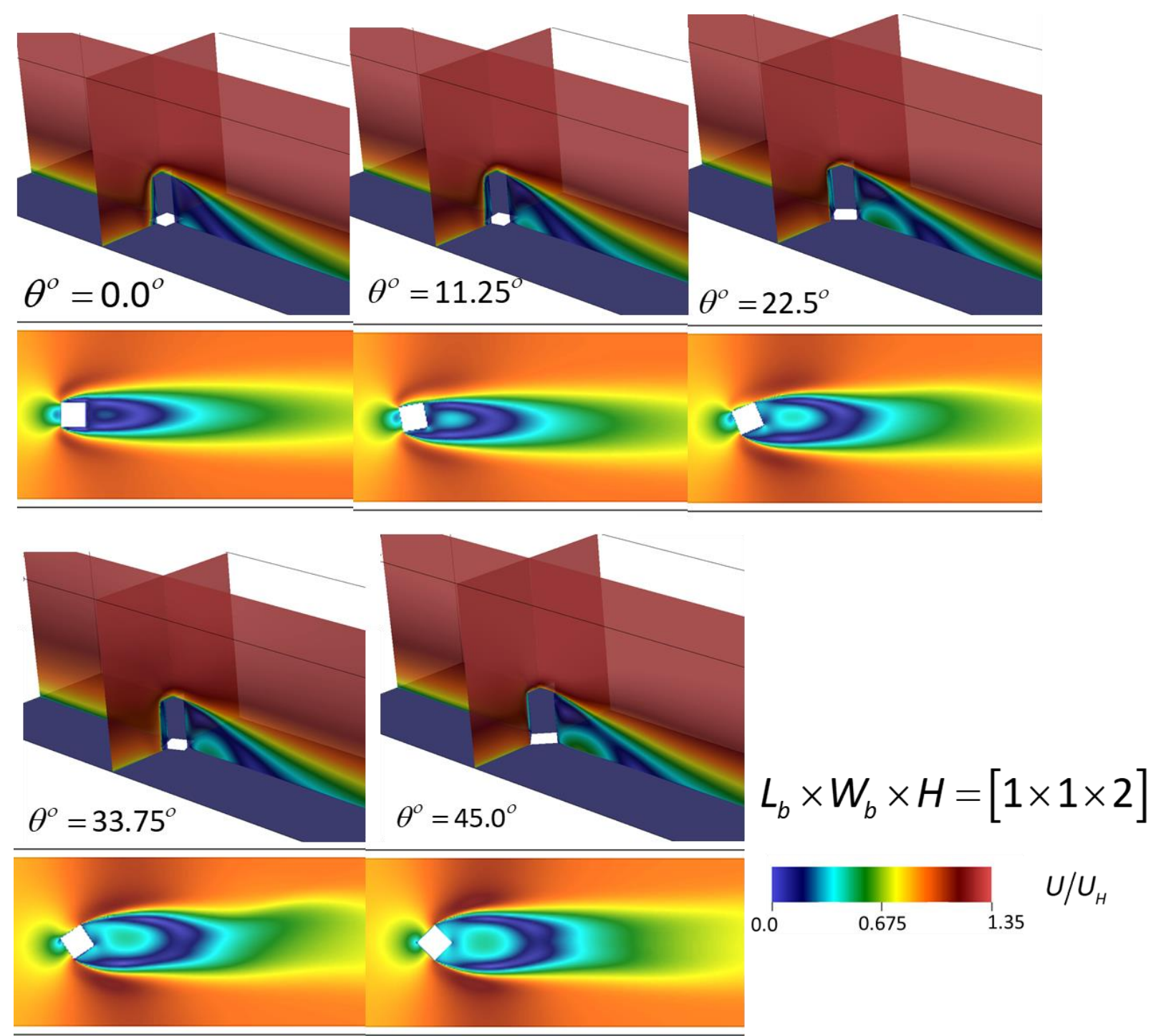

Figure 6 Wake results for $L_{b \times} W_{b} \times H=[1 \times 1 \times 2]$ for various angles of wind attack (Top: perspective view, Bottom: slide at $\mathrm{z}=0.1 \mathrm{H}$ ) 

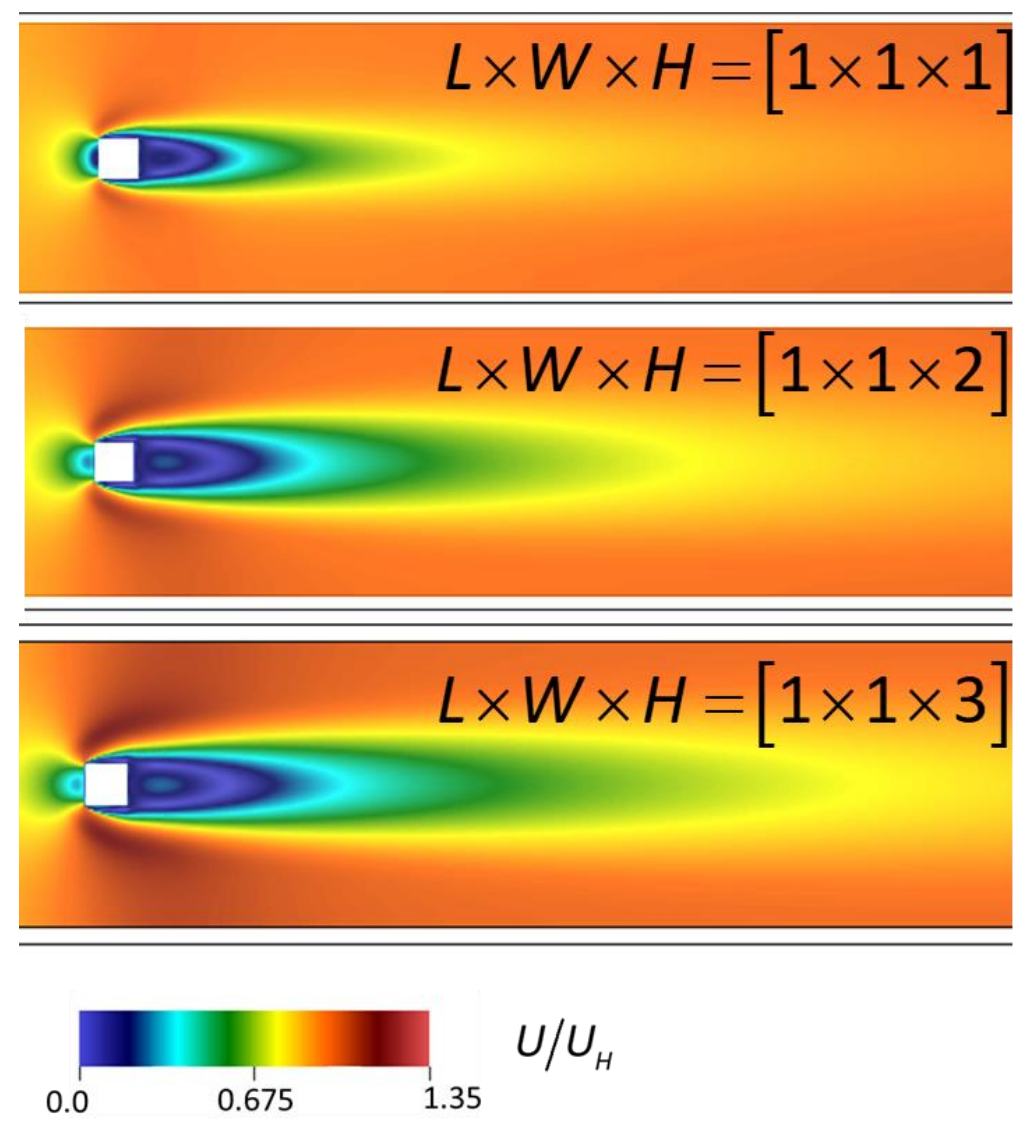

Figure 7 Wake results for various height at a slide at $z=0.1 \mathrm{H}$.

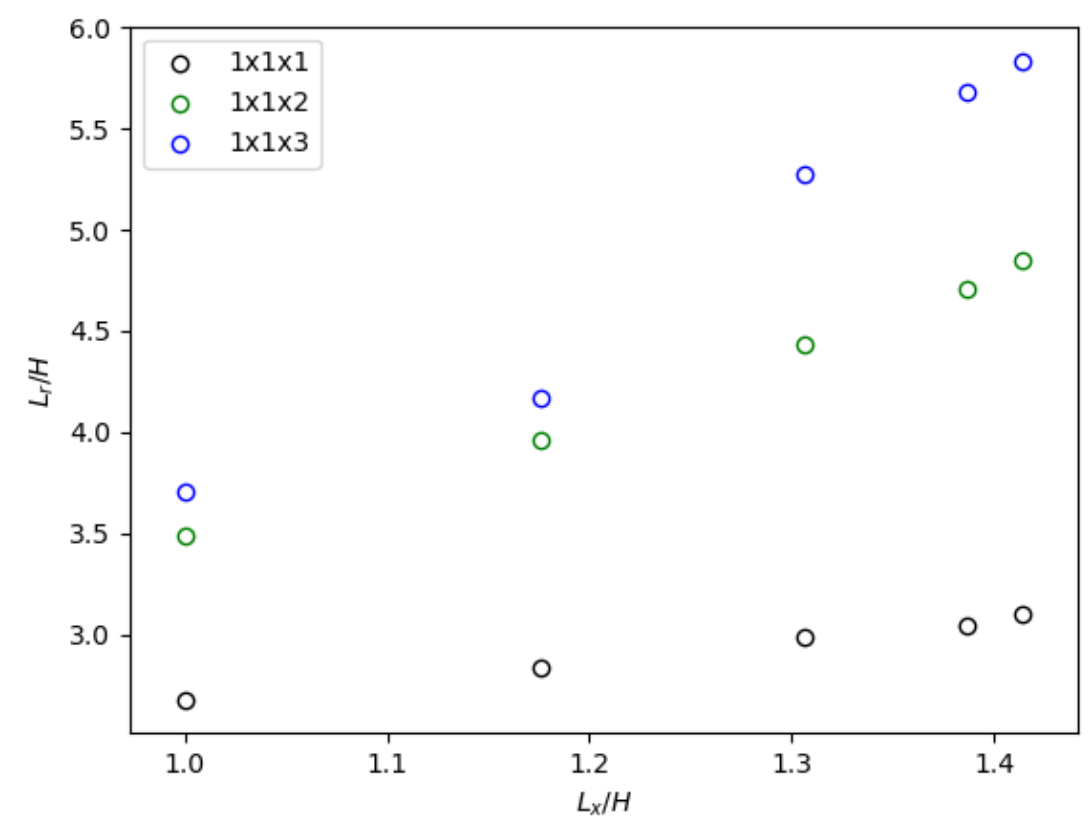

Figure 8 Effect of the streamwise length $L_{x}$ (due to different angle of attack) and the height of the building on the recirculation length $L_{r}$. 


\subsection{Low Order Model Development}

\subsection{Low Order Model Formulation}

A generalization of the low order model (LOM) based on the analytical threedimensional model by Robins and Apsley (2020) based on Counihan, Hunt and Jackson (1974). According to the analysis by Counihan et al. (1974), Hunt and Robins (1982) and Robins and Apsley (2020) (also see Apsley, 1988) the following wake perturbation analytical expression can be derived from the momentum equations:

$$
f=\frac{\Delta u}{U_{H}}=1-\alpha\left[\frac{W}{\lambda_{y}}\right]\left[\frac{H}{\lambda_{z}}\right]^{2} f(\xi) h(\eta)
$$

where $\Delta u=U(x, y, z)-U\left(x_{\text {Ref }}, y_{\text {Ref }}, z\right), U_{H}$ is the velocity at the height of the building, $x_{\text {Ref }}=-4.5 H$ and $y_{\text {Ref }}=0.0, W$ and $H$ are the width and height of the building and $a$ is the wake strength parameter. $\lambda_{y}$ and $\lambda_{z}$ are the characteristic wake zone thicknesses in the spanwise and vertical direction as they are shown in figure (Figure 8). $\lambda_{y}$ and $\lambda_{z}$ are computed as:

$$
\begin{aligned}
& \lambda_{y}(x)=\left(\frac{D_{y}\left(x-x_{o}\right)}{U_{H}}\right)^{1 / 2} \\
& \lambda_{z}(x)=\left(\frac{D_{z}\left(x-x_{o}\right)}{U_{H}}\right)^{1 / 2}
\end{aligned}
$$

where eddy viscosities $D_{y}$ and $D_{z}$ are the spanwise and vertical diffusivities of the wake (eddy viscosities), and $x_{o}$ is the virtual origin of the wake's gaussian. The functions $f(\xi)$ and $h(\eta)$ are computed as:

$$
\begin{aligned}
& f(\xi)=\frac{\xi}{2} \exp \left(\frac{-\xi^{2}}{4}\right) \\
& h(\eta)=\left(\frac{1}{2 \sqrt{\pi}}\right) \exp \left(\frac{-\eta^{2}}{4}\right)
\end{aligned}
$$

where $\xi=\frac{z}{\lambda_{z}}$ and $\eta=\frac{y}{\lambda_{y}}$.

A similar model has also been proposed by Kothari et al. (1979) and Peterka et al. (1985) who observed that simple wake models cannot capture the effect of accelerated flows around the buildings. For this reason, Kothari et al. (1979) and Peterka et al. (1985) proposed an additional term to account for the acceleration effect due to the formed horseshoe vortex upstream from the building. The general form of such a correction for the wake equation is according to Hunt (see Peterka et al. 1985): 


$$
f^{\prime}=\left.\frac{\Delta u}{U_{H}}\right|_{\text {due to HV }}=\Gamma y^{\prime} h x\left(\frac{1}{\left(y^{\prime 2}+h^{2}+z^{2}\right)^{2}-4 z^{2} h^{2}}\right)
$$

where $y^{\prime}=y_{v}-y . y_{v}$ and $h$ are the distances to the center of the horseshoe vortex and $\Gamma$ is the circulation at $x=0.0$ (Peterka et al. 1985).

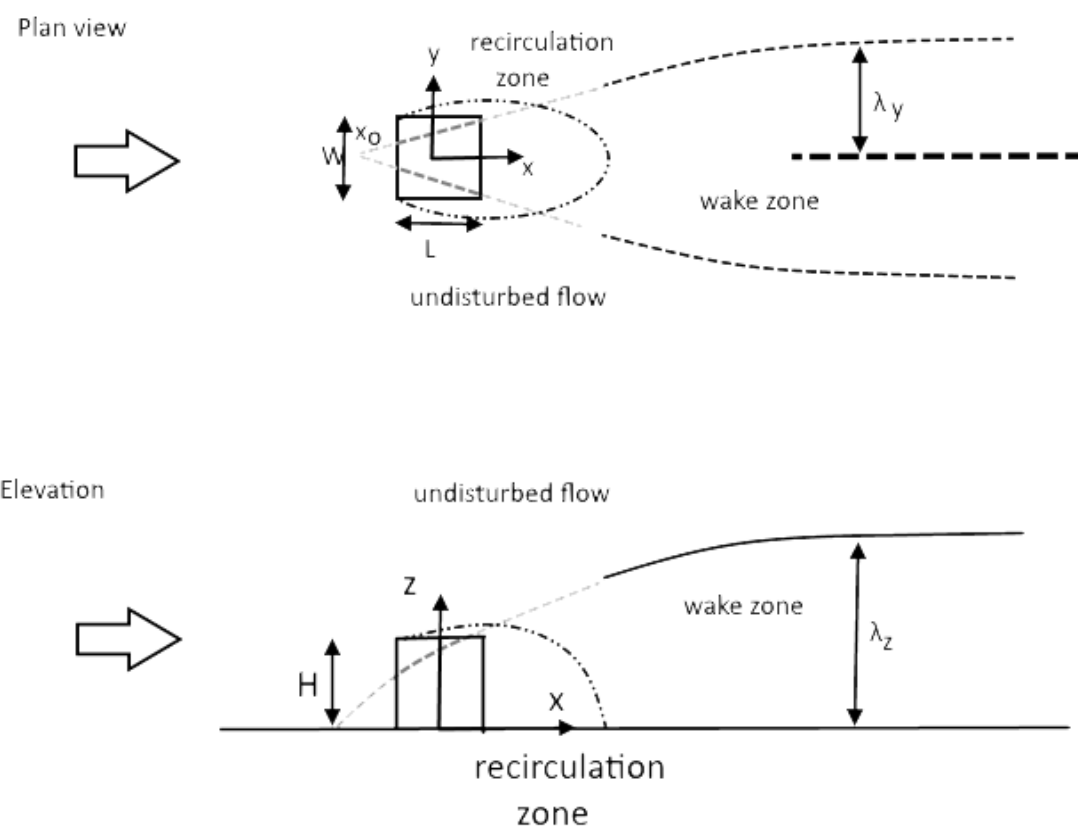

Figure 9 Wake zone length scales $\lambda_{y}$ and $\lambda_{z}$ (adopted from Robins and Apsley 2020).

The equations above have been derived and used for the case of a single cuboid and for wind angle of attach zero (flow perpendicular to the face of the cube). In the present analysis the analytical equations presented above have been generalized for various angles of attack by means of adjusting the regression parameters $\alpha, D_{y}, D_{z}$, and $x_{o}$ for the main wake model and $\Gamma, y_{v}$ and $h$ for the horseshoe-vortex/accelerated flow correction.

The low order model parameters for various angles of attack and building aspect ratios (see table 1 for the full list of cases) were estimated using the surrogate model technique combined with the machine learning based algorithms included in the open-source package Tensorflow (Abadi et al. 2015). The Neural Network architecture consists of 4 branches for the main wake model component of the LOM and 3 branches for the wake correction of the LOM. The parameters $x_{o}$ and $\alpha$ were assumed to be functions of $H, L$ and $W$ while the eddy diffusivities $D_{y}$ and $D_{z}$, were assumed to be functions of $H, L, W$ and $x, y, z$. For the horseshoe-vortex/accelerated flow correction the parameters $\Gamma, y_{v}$ and $h$ were assumed to be functions of $H, L$, and $W$. Positivity preserving constraints were embedded in the model as per the physical range of parameters (e.g. eddy 
viscosities $D_{y}$ and $D_{z}$ cannot get negative values). The number of layers/neurons/activations and optimization hyperparameters were manually tuned to improve the robustness and accuracy of the LOM. $70 \%$ of the data presented in paragraph 2 were used for the training and validation of the model and $30 \%$ for the testing of the model.

The accuracy of the trained model to predict the testing data can be seen in figure 10 . The trained model results for various locations $(x=5 \mathrm{H}, 6 \mathrm{H}, 8 \mathrm{H}$ and $10 \mathrm{H})$ are shown in figure 11. The effect of the horseshoe-vortex/accelerated flow correction is shown in figure 12. In agreement with the observation by Kothari et al. (1979) and Peterka et al. (1985) a simple wake model seem to be unable to capture the acceleration effects. Improved results can be observed when a correction is introduced. Finally, the comparison between CFD results and the prediction of the LOM for $x=3 \mathrm{H}, 5 \mathrm{H}, 6 \mathrm{H}, 8 \mathrm{H}$ and $10 \mathrm{H}$ is shown in figure 13. In general, the predictions of the proposed LOM are in good agreement with the CFD results.

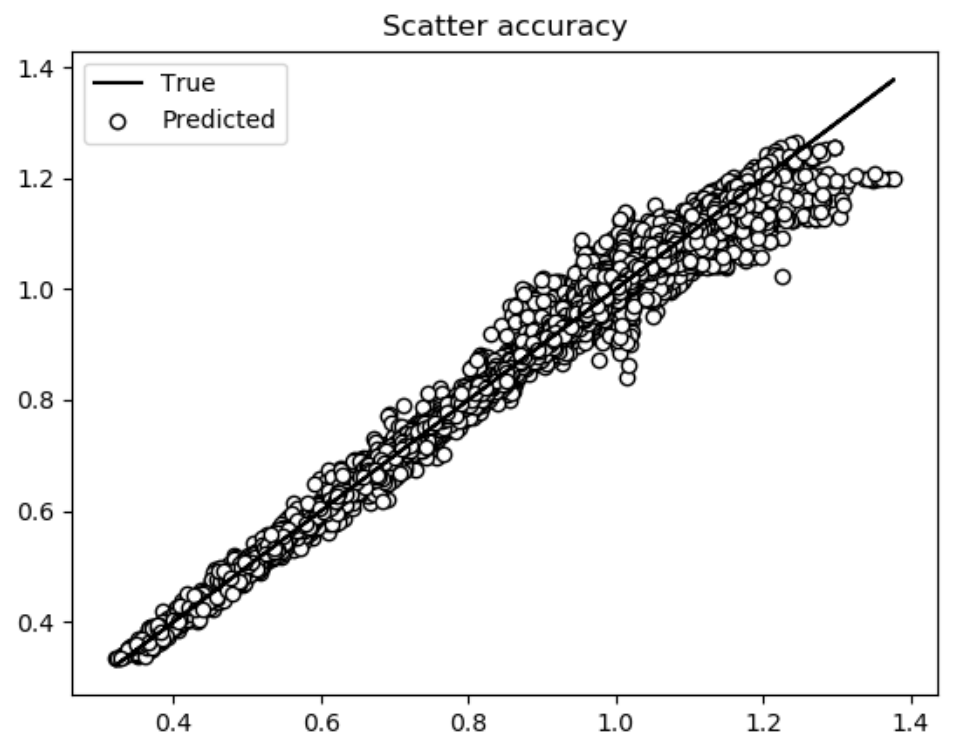

Figure 10 Accuracy of the trained LOM against testing data. 


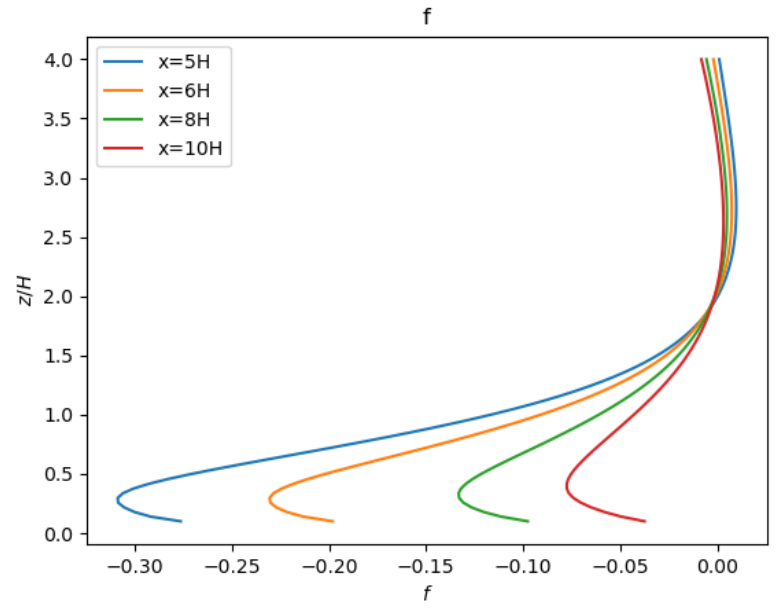

Figure 11 LOM prediction for various downwind locations at the centerline $(y=0.0)$ for the case of $L_{b} \times W_{b} \times H=[1 \times 1 \times 1]$.
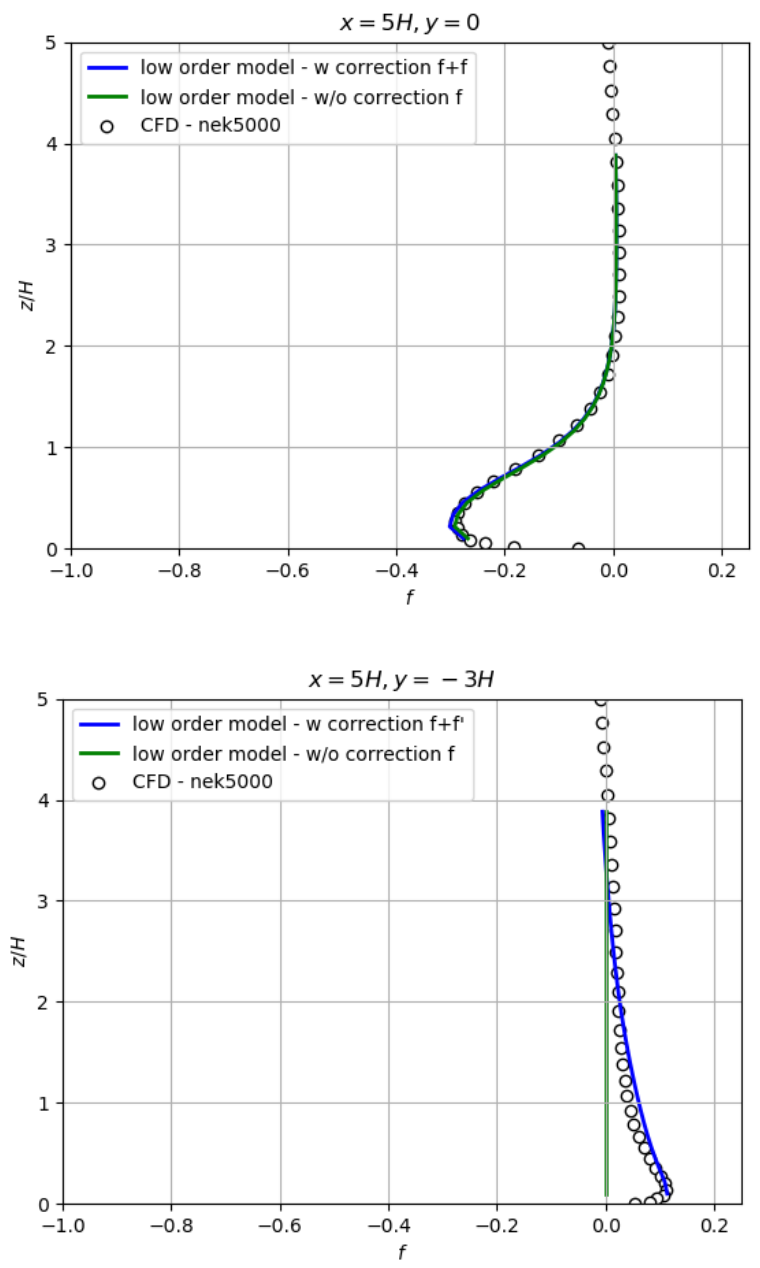

Figure 12 Effect of horseshoe-vortex/accelerated flow correction on the LOM results. Comparison against CFD results for $x=5 H$ at the centerline $(y=0.0)$ and at $\mathrm{y}=-3 \mathrm{H}$ for the case $L_{b} \times W_{b} \times H=[1 \times 1 \times 1]$. 

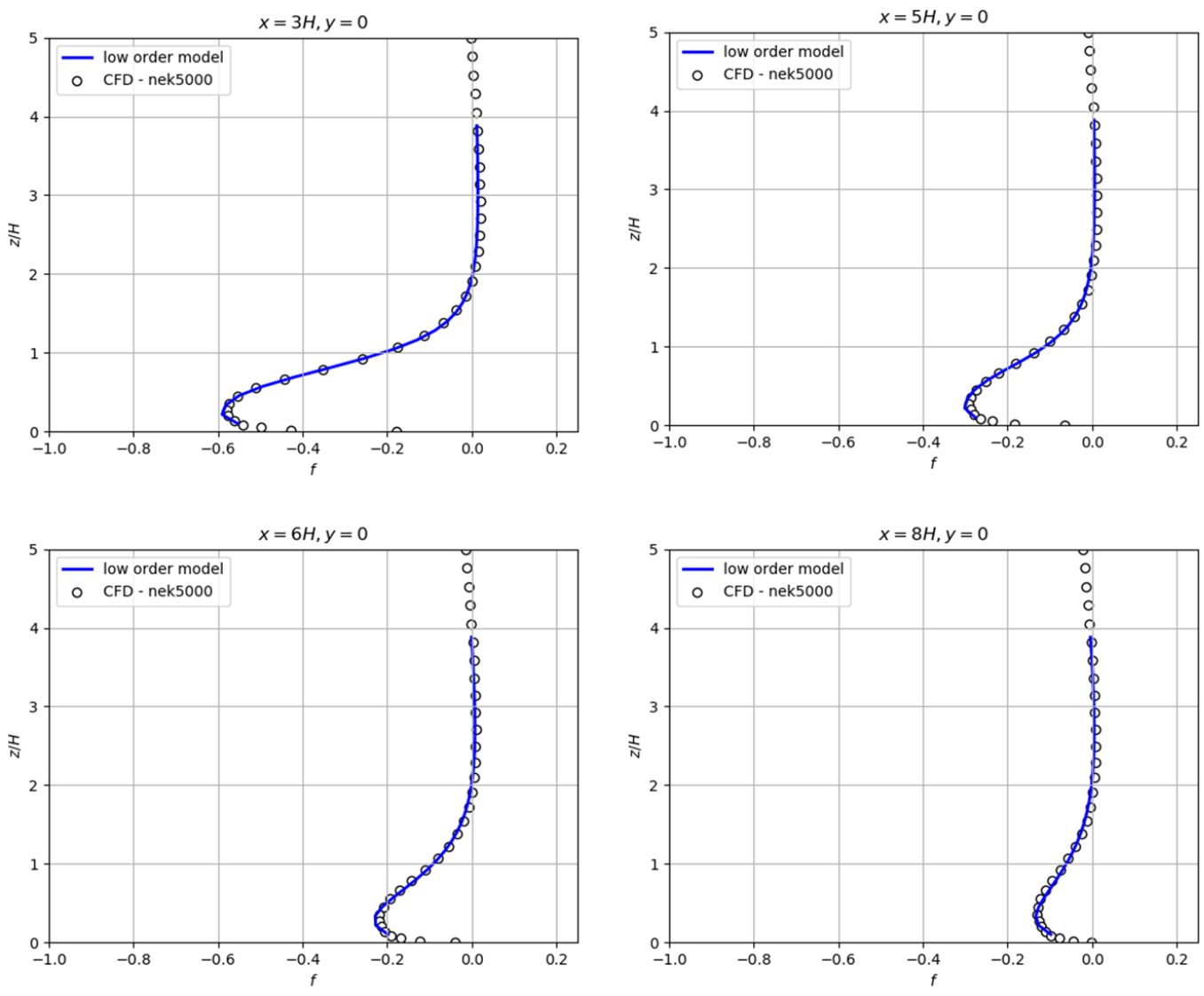

Schematic of plan view:

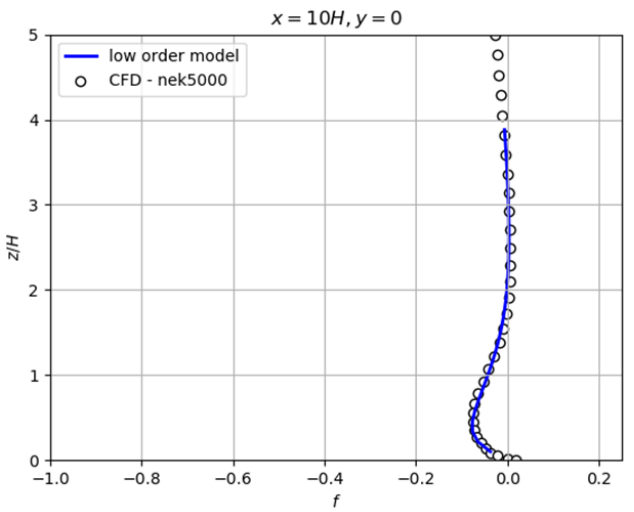

Flow directions

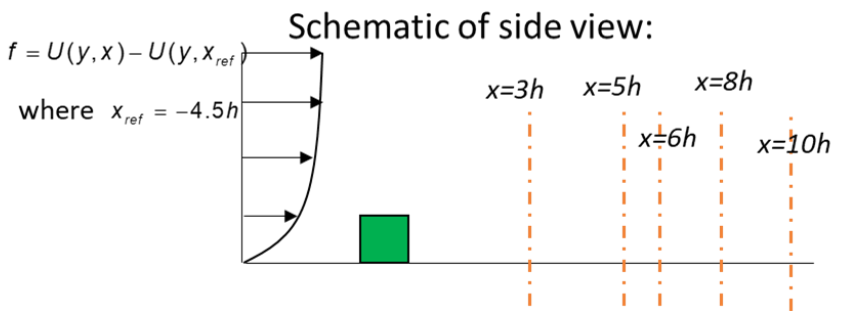

Figure 13 Comparison of LOM results against CFD results at 5 characteristic locations at the centerline $(y=0.0)$ for the case of $L_{b \times} W_{b} \times H=[1 \times 1 \times 1]$. 


\subsection{Low Order Model availability}

The low order model described here in has become available on Github: https://github.com/NREL/dw-tap-lom-anl

\subsection{Acknowledgement}

This work is supported by the Department of Energy's (DOE) Energy Efficiency and Renewable Energy (EERE) Wind Energy Technologies Office (WETO) under contract No. PRJ1007182 Tools Assessing Perf 35173. This research used resources of the Argonne Leadership Computing Facility, which is a DOE Office of Science User Facility supported under Contract DE-AC02-06CH11357. We gratefully acknowledge the computing resources provided on Bebop, a high-performance computing cluster operated by the Laboratory Computing Resource Center at Argonne National Laboratory.

\subsection{References}

Abadi M. , Agarwal A., Barham P., Brevdo E., Chen Z., Citro C., Corrado, G. S., Davis, Dean, A. J., Devin, M. , Ghemawat, S., Goodfellow, I., Harp, A., Irving, G., Isard, M., Jozefowicz, R. ..., and Zheng, X. (2015). Tensorflow: Large-scale machine learning on heterogeneous distributed systems. arXiv preprint arXiv:1603.04467.

Apsley, D. D., 1988, A model for dispersion in the wake of large buildings, CEGB Report $\mathrm{RD} / \mathrm{L} / 3359 / \mathrm{R} 88$

Counihan, J. J. C. R., Hunt, J. C. R., and Jackson, P. S. (1974). Wakes behind twodimensional surface obstacles in turbulent boundary layers. Journal of Fluid Mechanics, 64(3), 529-564.

Hunt, J.C.R. and Robins, A.G. (1982). A model for assessing dispersion of plumes from sources in the vicinity of cuboid shaped buildings, Proc. EUROMECH Conference on Surface Mounted Bluff Bodies in Turbulent Boundary Layers, Lisbon, Portugal.

Kothari, K. M., Peterka, J. A., and Meroney R. N. (1979). Stably stratified building wakes." Colorado State University.

Peterka, J. A., Meroney, R. N., and Kothari, K. M. (1985). Wind flow patterns about buildings. Journal of Wind Engineering and Industrial Aerodynamics, 21(1), 21-38.

Robins A. G. and Apsley D. D. (2020). Modelling of building effects in ADMS http://www.cerc.co.uk/environmental-software/assets/data/doc_techspec/P16_01.pdf (access 03/20/2021)

Fischer, P. F., Lottes, J. W., and Kerkemeier, S. G. (2008). "Nek5000 Web page", http://nek5000.mcs.anl.gov (accessed 01/30/2021)

Speziale, C. G., Abid, R., \& Anderson, E. C. (1992). Critical evaluation of two-equation models for near-wall turbulence. AIAA journal, 30(2), 324-331. 
Snyder, W. H., and Lawson R. E. (1994). Wind-tunnel measurements of flow fields in the vicinity of buildings. U.S. Environmental Protection Agency, Washington, D.C., EPA/600/A-93/230 (NTIS PB93236594). 
Argonne

\section{Computational Science Division}

Argonne National Laboratory

9700 South Cass Avenue, Bldg. 240

Argonne, IL 60439

\section{Environmental Science Division}

Argonne National Laboratory

9700 South Cass Avenue, Bldg. 240

Argonne, IL 60439

www.anl.gov

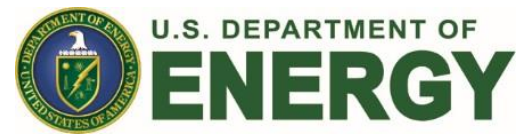

Argonne National Laboratory is a U.S. Department of Energy laboratory managed by UChicago Argonne, LLC 